\title{
Applying Quality Function Deployment in Game Design
}

\author{
Yi Wang \\ Department of Fashion Design, business and Management \\ School of materials \\ University of Manchester, UK \\ Yi.wang-2@manchester.ac.uk
}

\begin{abstract}
Quality Function Deployment is a useful tool in product development and design with the application of voice of customer in collecting customers' needs. The purpose of these studies is to apply the effective use of Voice of Customer in redesigning a game for educating youth about cyberbullying. The data collection from Voice of Customer or consumers' feedback might help the producer to improve the quality of the designed game. The Voice of Customer provides the needs of redesign, based on users' requirements. In game designing phase, it is very important to distinguish each specification and characteristic, which translated from Voice of Customer to House of Quality matrix. This research would indicate the customers' requirements and production team idea might be measured and translated to a systematic data. The specific technical data could be planned ahead with specific design details 1 . As a conclusion, this study would benefit the Small Medium Enterprises design team or the designers to style an item from customers view with organised projection of the product. The finding also could assist designers to recognise specific quality details of the education from consumers to make further detail research from the customers' requirements.
\end{abstract}

Keywords-house of quality; voice of customer; educational game design; quality function deployment

\section{INTRODUCTION}

Simulation games have grown in use as a training and education tool over the last fifty years. The main aim in this research is to apply QFD method in the redesign educational game. This is to see whether this method is suitable. The first objective of this research is to utilise the application of Voice of Customer in redesigning. The User' feedback or known as the term 'Voice of Customer' could help the manufacturer to better the product before the following production. This Voice of Customer could offer a specific detailing for quality, which needs to be redesigned in a Game. It is crucial to identify each specification and characteristic, which translated from Voice of Customer to the House of Quality matrix. This matrix would help designers to design according to qualities that customer wants for a better and successful product in a market.

Quality functional deployment provided an efficient tool for translating the customers' voice into target engineering characteristics for a training game. Engineering quantity scales for the 'Hows' in the House of Quality and obtained targetengineering characteristics for the new game.

\section{LITERATURE REVIEW}

First, In the UK, almost all children aged between 8 and 17 years report using the Internet, with approximately $80 \%$ of households with children having Internet access [1]. Similar levels of Internet access are reported in Australia: $91 \%$ of households with children under 15 years have access to the Internet [2]. In the UK, $30 \%$ of 7 to 11 year olds reported having social networking accounts [3] and virtual world accounts [4]. There is also a high level of mobile phone access among children $[1,5]$. Monks, Ortega, Robinson, and Worlidge [6] found that $72 \%$ of youth in UK owned a mobile phone. $46 \%$ of 9 to 16 year olds owned a smartphone, with variability across countries; smartphone owner-ship was highest in Denmark compared with the UK, Italy, Ireland, Belgium, Portugal, and Romania [7].

Cyberbullying has been defined as "a form of intimidation, harassment, and mistreatment on the part of an individual or group towards another, which involves the use of technological means to channel the aggression repeatedly and involving an imbalance of power between the perpetrator and the target" [8].

Talwar, Gomez-Garibello, \& Shariff, [9] indicates the nature of technology, and consequently the nature of cyberbullying, is constantly changing, with different methods becoming more popular at different time points. Cybervictimization was related to increased social anxiety, even when controlling for experiences of traditional bullying [10]. Campbell, Spears, Slee, Butler, and Kift, [11] found that cyber victims reported higher levels of anxiety and depression than victims of traditional bullying. In a sample of 7 to 11 year olds in the UK, $5 \%$ self-reported as being an aggressor and $23 \%$ as a victim [8].

Simulation games used as learning tools is very popular in Europe and US [12]. The main requisites of any successful computer game are representation, interaction, conflict, and safety [13]. A game design is the set of type definitions and rules of a game, usually composed from several game modules [14]. It allows react to the player's actions quickly and appropriately [15]. There is no such thing as a "standard" game design rules. Design treatment is a brief discussion of the game unique features and target audience [16]. Most designers emphasize that game rules are formed at this stage, and the crucial moment is to make the rules to be consistent [17]. Learning by Playing is a concept describing the educational 
value of play as a part of informal learning. The aim is to gain knowledge about users' practice and experience of system use in order to achieve more effective understanding [18]. A good game should be designed by constructing various learning environments that are adaptive, scalable and flexible [19].

\section{QUALITY FUNCTION DEPLOYMENT}

Quality function deployment (QFD) was developed in Japan by Akao in 1972 as a way of matching preferences against engineering characteristics with the objective of systematically translating the 'voice of customer' [20 and 21]. At the early stage, the focus of QFD was more into engineering process. This concept spread in the US after the successful production of Japanese company in producing car; low on production cost with minimal complaints and feedbacks from customer [22].

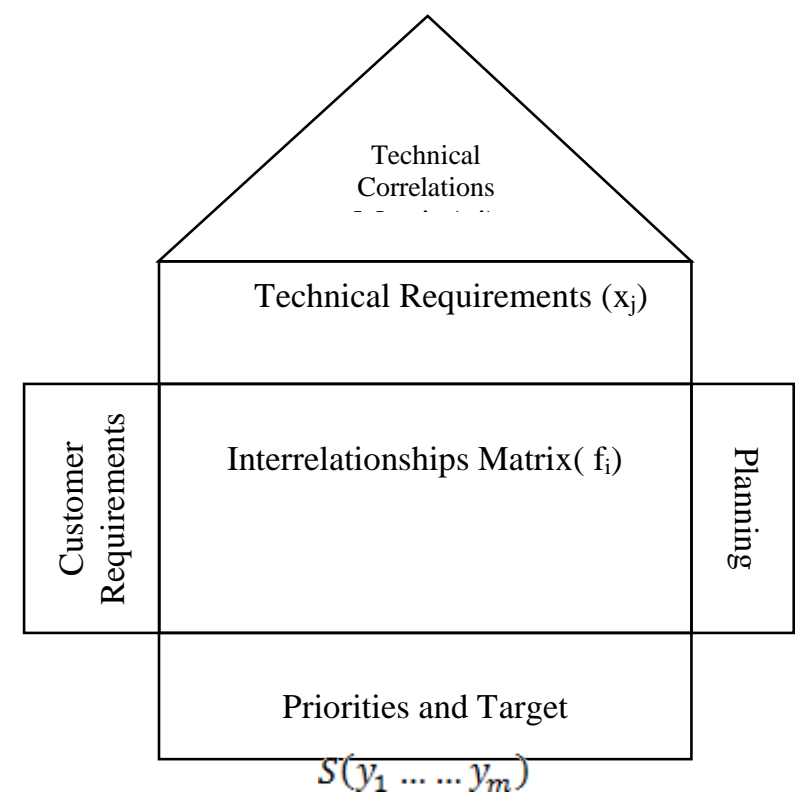

Fig. 1. Basic structure of the House of Quality.

QFD concept was applied to product development and production but not restricted to product design and engineering view but beginning to be implemented in marketing strategies, production planning, prototype evaluation, sales and services as well. QFD is normally been used in automotive industries in the decades back but now other industries are applying it as well. Apparel and textile industry is one of them.

The House of Quality (shown in Fig. 1), is one of the tools used for QFD. It is a formal articulation of engineering characteristics against performance characteristics or customer requirements. Determining the target values of engineering characteristics from the customer requirements is one of the essential outcomes of the QFD process [23]. The proposed optimisation approach attempts to address these problems, by using multi-attribute value theory, combined with linear regression and linear programming, to determine the target values of engineering characteristics in the QFD process [24]. $y_{i}=$ customer requirement on level of performance for performance characteristics $i$. (where $i=1, \ldots, m$ );

$x_{j}=$ Technical Requirement for engineering characteristic $j$. (where $j=1, \ldots, n$ );

$f_{i}=$ functional relationship between performance characteristic $\mathrm{i}$ and engineering characteristics, where $i=1, \ldots, m$. in other words $y_{i}=f i\left(x_{1}, \ldots, x_{m}\right)$;

$g_{j}=$ Technical correlations between engineering characteristic $j$ and other engineering characteristics, where, $j=1, \ldots ., n$.

The problem could be simplified into a conventional linear program as below:

1. Objectives:

Find target values $x_{1}, x_{2}, \ldots, x_{n}$, which maximise overall satisfaction for $S\left(y_{1}, \ldots, y_{m}\right) \dagger$.

$$
S\left(y_{1} \ldots \ldots y_{m}\right)=\sum_{i=1}^{m} w_{i} \mathrm{~F}_{i}\left(y_{i}\right)
$$

where $F_{i}\left(y_{i}\right)$ is the individual value function for performance characteristic $i$ and $w_{i}$ is the weight of performance characteristic $i$.

\section{Constraints:}

$$
\text { Subject to } y_{i}=. f_{i}\left(x_{1}, \ldots ; x_{m}\right) ; x_{j}=g_{j}\left(x_{1}, \ldots, x_{n}\right) \dagger \text {. }
$$

\section{DESIGN PROJECT}

The paper is based on BECYBERSAFE project, which is financed by ERASMUS + . The milestone that BECYBERSAFE set up this project comes from the European Commission, EU Kids Online Network (www.eukidsonline.net) has revealed an overall perspective on European kids' (9-16 ages) internet related experiences.

The research shows that $19 \%$ of children have been bullied either online or offline, and $12 \%$ bullied someone else. Considering the results of previous researches, BECYBERSAFE is looking to address this problem by making survey-based research in our partner countries among teenagers' online opportunities, risks, and safety implications against cyberbullying via a collaborative partnership in a supportive physical and social environment with the help of laws and policies against cyberbullying.

Through BECYBERSAFE, the partnership wants to create a set of approaches and strategies within the EU's vision based on different ways of motivating and engaging teenagers in safer use of internet and campaign against cyberbullying.

While one of our main aims is to reduce cyberbullying at schools, the other aims are taking precaution implementations against dropping out of schools and decreasing high levels of absenteeism. The students, who are bullied both in cyberspace and at school, experience difficulties at school such as low marks, poor concentration, and absenteeism.

Let: 

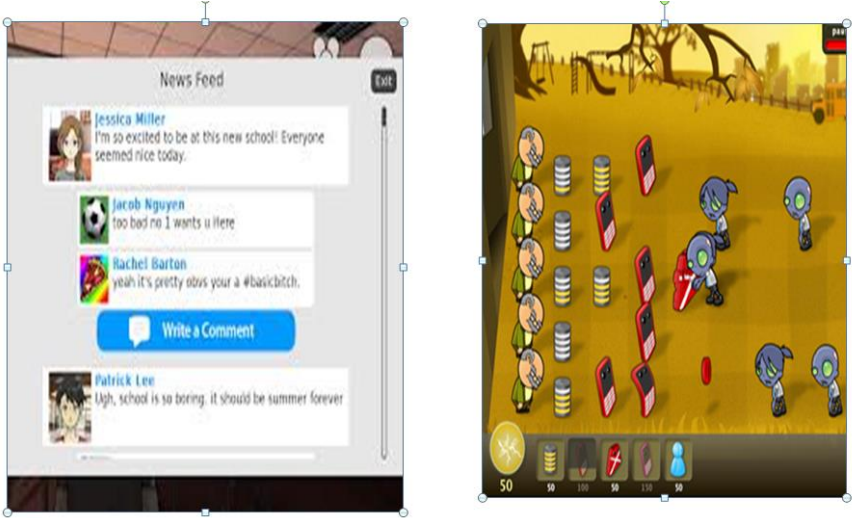

Fig. 2. Previous exsisting game.

There have been several existing serious games in the education of cyberbullying. Mostly fall into two categories as shown in Figure 1. One is textual based and the other is none textual. It is clear from the initial feedback from 10 groups of pupil from 6 European countries that the textual is too boring.

The designers find also that the none-textual game can be too entertaining and emphasis more on the game experience rather than educational purposes.

With this project, the partnership is planning to raise awareness of the society and to provide core knowledge and guidelines which can help students, parents, and teachers for avoiding the undesired results of cyberbullying.

The partnership will also develop a BECYBERSAFE ICT tool (A game), a Guide Book to be implemented during the project among school directors, parents, teachers and the students, providing numerous anti-cyberbullying activities, practical advice and information on how to prevent it. This paper only focuses on the development of the Game.

Table 1 HOQ

\begin{tabular}{|l|l|l|l|l|l|l|l|}
\hline & Weight & instruction & Reliability & Al & Realism & Educational & Difficulty \\
\hline Easy to play & 5.0 & 5 & & & & 2 & \\
\hline Adaptation & 5.0 & & 2 & & & 2 & \\
\hline Interesting & 5.0 & & & & 7 & & 5 \\
\hline Graphic & 4.0 & & & & 5 & & \\
\hline Challenging & 6.0 & & & 10 & & & \\
\hline Variety & 3.0 & & & 3 & & & 3 \\
\hline & & 25 & 10 & 69 & 55 & 20 & 34 \\
\hline
\end{tabular}

After data was obtained and translated as voice of customer, a selected panel went through a set of survey. Customer requirements or customer attributes were listed according to the rank order design [25]. The rank order design is determined by the importance mean weightage. The higher the value means the higher preference of the customer towards the attributes, which reflect the taste towards the existing and hypothetical product [23 and 26].

The number meaning in the HOQ are: 7-10 represents the strong relationship, 4-6 values as the moderate relationship, meanwhile 1-3 categorises the weak relationship in the HOQ, as shown in Table 1 The strong relationship of consumer requirements and technical requirements should be considered when redesigning new games are the source of game and finishing has strong relationship which should considered. The "roof" shows the possible conflicts in the technical requirements. The numbers 9 represents strong relationship, 5 represent medium relationship while 3 represents weak relationship are used to identify the possible conflicts.

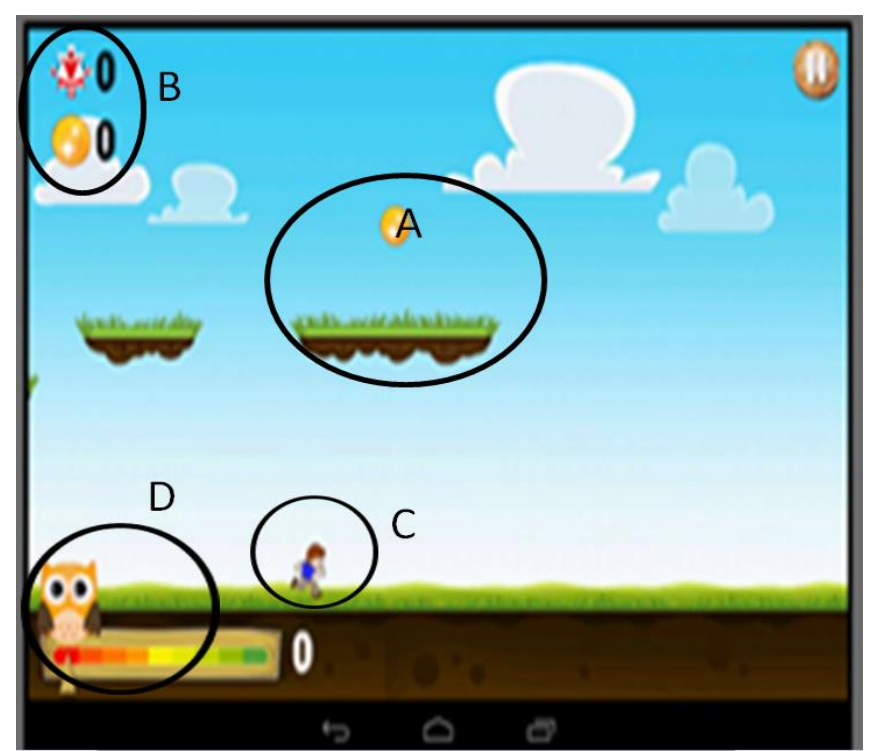

Fig. 3. The final game.

The final game is shown in Figure 3. It is designed for androids and applet but can be played on other platform using an emulator.

In Fig. 3:

A) Represent the platform where the controlled character can jump on. On some platform there are coins to be collected. On rare occasion there will be a red token.

B) The numbers indicate how many coins and red token the players have collected.

C) This is the playable character. In the beginning of the game the sex of the character can be selected. The character can only move forward and jump.

D) The bar indicated the progress. At end of each level, a question is appeared regarding cyberbullying. The player is also presented with a multiple choice answer. The number indicates how many question the player has answered correctly. For each wrong answer a owl will appear to provide the correct one.

\section{CONCLUSION}

This research focus on how QFD is applied in Educational game design. Quality and creativity of a product are vital in producing a product. Nevertheless, the Educational game has 
some characteristics that need to be retained and some should be improved to make it competitive clothing choice for Users.

In order to achieve this, QFD is applied to identify the users' needs and analyse which characteristics are important in a product and which should be improved developed or eliminated. This could be beneficial for the educators. QFD could help the designers to understand the product itself from customers view. They could actually plan the product specifications from the technical side and creative design motifs before it is being execute physically. This could save cost, time and to generate more ideas on design base on one HOQ as a blueprint.

\section{REFERENCES}

[1] Byron Review, Safer children in a digital world, 2008. (http://webarchive.nationalarchives.gov.uk/20130401151715), (http://www.education.gov.uk/publications/eOrderingDownload/DCSF00334-2008.pdf)

[2] Australian Bureau of Statistics, Children of the digital revolution, 2011. (http://www.abs.gov.au/socialtrends)

[3] H. Broadbent, L. Fell, P. Green, \& W. Gardner, Have your Say: Listening to young people about their online rights and responsibilities. Plymouth, UK: Childnet International and UK Safer Internet Centre, 2013. (http://www.saferInternet.org.uk/research)

[4] D. Holloway, L. Green \& S. Livingstone, Zero to Eight Young children and their Internet use, 2013. (http://www.eukidsonline.net)

[5] Mobile Life Youth Report, The impact of the mobile phone on the lives of young people, 2006.

(http://iis.yougov.co.uk/extranets/ygarchives/content/pdf/CPW06010100 42.pdf)

[6] C. P. Monks, R. Ortega, S. Robinson \& P. Worlidge, "Cyberbullying among primary school-aged pupils,” Kwartalnik Pedagogiczny, Vol. 4, pp. 167-181, 2009.

[7] G. Mascheroni and K. Ólafsson, "The mobile Internet: access, use, opportunities and divides among European children," New Media and Society. Advance online publication, 2015.

[8] C. P. Monks, J. Mahdav and K. Rix, "The emergence of cyberbullying in childhood: Parent and teacher perspectives," Psicología Educativa, Vol. 22, pp. 39-48, 2016.

[9] V. Talwar, C. Gomez-Garibello and S. Shariff, Adolescents' moralevaluations and ratings of cyberbullying: The effect of veracity and inten-tionality behind the event. Computers in Human Behavior, Vol. 36, pp.122- 128. 2014.

(http://dx.doi.org/10.1016/j.chb.2014.03.046Vandebosch)

[10] J. Juvonen \& E. F. Gross, "Extending the school grounds? - Bullying experiences in cyberspace," Journal of School Health, Vol. 78, pp. 496505, 2008.
[11] M. Campbell, B. Spears, P. Slee, D. Butler \& S. Kift, "Victims" perceptions of traditional and cyberbullying, and the psychosocial correlates of their victimisation," Emotional and Behavioural Difficulties, Vol. 17, pp. 389-401, 2012

[12] A. Lopez, "Pretending for real: Simulation Games are gaining popularity as educational tools in fields ranging from humanitarian action to business management and accident prevention", 1999. (http://www.unesco.org/courier/1999 04/uk/apprend/txt1.html)

[13] S. Mukherjee, "The Spy in the Computer, A study of how computer games, as a modern narrative form, draw on and develop the tradition of espionage fiction”, December 2004. Jadavpur University, Calcutta 2004.

[14] Glossary - Designing Games with Xcong, 2005. (http://sources.redhat.com/xconq/manual/xcdesign_60.html)

[15] G. Howland, “2D vs. 3D: Fight of the Century?” September 5, 1998. (http://www.lupinegames.com/articles/2dvs3d.htm)

[16] F. D. Laramee, “The Game Design Process,” November 1999. (http://www.gamedev.net/reference/articles/article273.asp)

[17] W. Kramer, "What Makes a Game Good?" The Games Journal. July 2000 (http://www.thegamesjournal.com/articles/WhatMakesaGame.shtml)

[18] S. Wilson, M. Bekker, P. Johnson \& H. Johnson, Helping and Hindering User Involvement - A Tale of Everyday Design. HCI97 Electronic Publications. Queen Mary and Westfield College. London, UK, 1997. (http://sigchi.org/chi97/proceedings/paper/sw-obf.htm)

[19] S. Thomas, G. Schott and M. Kambouri, "Designing for Learning or Designing for Fun? Setting Usability Guidelines for Mobile Educational Games", Proceedings of MLEARN 2003: Learning with Mobile Devices, London, 2003.

(http://www.ioe.ac.uk/hgm/research/SkillsforLife/MobileLearningPrinci ples.pdf)

[20] Y. Akao, Quality Function Deployment: Integrating Customer Requirements into Product Design, Productivity Press, Cambridge, Mass, 1990

[21] J. L. Bossert, Quality Function Deployment: A Practitioner's Approach, American Society for Quality, Milwaukee, USA, 1990.

[22] L. Cohen, Quality function deployment How to make QFD work for you. Reading, Mass.: Addison-Wesley, 1995.

[23] J. R. Hauser, \& D. Clausing, The house of quality. Harvard Business Review, 1988

[24] L. K. Chan \& M. L. Wu, "A systematic approach to quality function deployment with a full illustrative example," Omega, Vol. 33, No. 2, pp. 119-139. (doi:10.1016/j.omega.2004.03.010), 2005.

[25] A. Griffin and J. Hauser, The Voice of the Customer. Marketing Science, Vol. 12, No. 1, pp. 1-27, 1993. (http://6-sigma-us.com/wp-content/uploads/2013/12/A-0101-2301.pdf)

[26] S. B. Han, S. K. Chen, M. Ebrahimpour \& M. S. Sodhi, "A conceptual QFD planning model," International Journal of Quality \& Reliability Management, Vol. 18, No. 8, pp. 796-812, 2001 